\title{
Uso de fitoterápicos como alternativa para a diminuição da sintomatologia
}

\section{recorrente na gravidez}

\author{
Use of phytotherapy as an alternative to reduce recurrent symptomatology in pregnancy \\ Uso de fitoterapia como alternativa para reducir la sintomatología recurrente en el embarazo
}

Recebido: 17/02/2021 | Revisado: 24/02/2021 | Aceito: 01/03/2021 | Publicado: 08/03/2021

Felipe Santos Rocha

ORCID: https://orcid.org/0000-0003-0749-3189 Universidade Tiradentes, Brasil E-mail: liperocha1999@gmail.com

Jordana Vitória Carvalho Santos

ORCID: https://orcid.org/0000-0001-9478-5707 Universidade Tiradentes, Brasil

E-mail: jordanavcarvalho@gmail.com

Rafaela Oliveira Resende

ORCID: https://orcid.org/0000-0001-9313-1648

Universidade Tiradentes, Brasil

E-mail: rafaela.florzinharesende@hotmail.com

Tatiane Batista dos Santos

ORCID: https://orcid.org/0000-0002-5807-4614 Universidade Tiradentes, Brasil

E-mail: tatiane0906@hotmail.com

Enoque Chaves de Almeida Junior

ORCID: https://orcid.org/0000-0001-6667-9579 Universidade Tiradentes, Brasil

E-mail: junioralmeida4888@gmail.com

Daniela Droppa Almeida

ORCID: https://orcid.org/0000-0002-8154-1030

Universidade Tiradentes, Brasil

E-mail: danieladroppa@gmail.com

Deyse Mirelle Souza Santos

ORCID: https://orcid.org/0000-0001-8310-2448

Universidade Tiradentes, Brasil

E-mail: deysemirellle@hotmail.com

Fernanda Costa Martins Gallotti

ORCID: https://orcid.org/0000-0002-9063-1273

Universidade Tiradentes, Brasil

E-mail: Fernanda.gallotti@ souunit.com.br

\begin{abstract}
Resumo
Os extratos naturais de plantas vêm sendo utilizados com finalidades farmacológicas há milhares de anos. Seu uso na medicina popular foi propagado de cultura para cultura e descrito nas diversas literaturas. Para tanto, buscou-se analisar os benefícios da utilização de fitoterápicos frente aos sinais e sintomas recorrentes do período gravídico. Trata-se de uma revisão integrativa de literatura, de cunho descritivo com abordagem qualitativa. Para o alcance dos resultados, foram analisados os resumos de 284 artigos disponíveis na íntegra, assim como, patentes obtidas a partir do cruzamento entre os descritores. Destes, 26 estudos foram selecionados por responderem a pergunta norteadora: " $O$ uso de fitoterápicos pode auxiliar no controle de sinais e sintomas advindos da gravidez?", seguindo-se assim para a continuidade da pesquisa. Após leitura dos estudos, identificou-se que 29 espécies de plantas são utilizadas como fitoterápicos para o tratamento de sintomas recorrentes na gestação, destacando-se o gengibre, a camomila, a hortelã e o alho. Quanto à prospecção tecnológica, foram selecionadas seis patentes que, em sua discussão relataram o potencial farmacológico do gengibre, melancia, dentre outras, frente ao tratamento das sintomatologias comuns na gestação. Dessarte observa-se que apesar do crescimento progressivo da importância dos medicamentos fitoterápicos, poucos estudos foram realizados com o intuito de se comprovar a eficácia da sua utilização frente aos sintomas característicos do período gravídico, sendo que inúmeras plantas medicinais ainda são aplicadas a partir do conhecimento popular bem empregado, ressaltando assim a importância do tema abordado.
\end{abstract}

Palavras-chave: Atenção primária à saúde; Cuidado pré-natal; Fitoterapia; Gravidez. 


\begin{abstract}
Natural plants extracts have been used for pharmacological purposes for thousands years. The use in popular medicine has been propagated from culture to culture and described in different literature. Therefore, sought to analyze the benefits of using herbal medicines in view of the recurrent signs and symptoms of the pregnancy period. It is an integrative literature review of a descriptive nature with a qualitative approach. To achieve the results, the abstracts of 284 articles available in full were analyzed as well as patents obtained based on that, the descriptors were crossed. Among these, 26 studies were selected for answering the guiding question: "Can the use of herbal medicines help in the control of signs and symptoms arising from pregnancy?". After reading the studies it was identified that 29 species of plants are used as herbal medicines for the treatment of recurrent symptoms during pregnancy especially the ginger, chamomile, mint and garlic. As for technological prospecting six patents were selected which, in their discussion, reported the pharmacological potential of ginger, watermelon, among others in the treatment of common symptoms during pregnancy. In this way, it is observed that despite the progressive growth in the importance of herbal medicines. Dessarte noted that the progressive growth in the importance of herbal medicines. A few studies have been carried out in order to prove the effectiveness of their use in face of the characteristic symptoms of the pregnancy period and that numerous medicinal plants are still applied from the knowledge well-employed.
\end{abstract}

Keywords: Primary health care; Prenatal care; Phytotherapy; Pregnancy.

\title{
Resumen
}

Los extractos de plantas naturales se han utilizado con fines farmacológicos durante miles de años. Su uso en la medicina popular se ha propagado de una cultura a otra y se ha descrito en diversas publicaciones. Para ello se buscó analizar los beneficios del uso de medicamentos a base de hierbas en vista de los signos y síntomas recurrentes del período de embarazo. Se trata de una revisión de la literatura integradora, de carácter descriptivo con enfoque cualitativo. Para lograr los resultados se analizaron los resúmenes de 284 artículos disponibles en su totalidad así como patentes obtenidas del cruce entre los descriptores. De estos, se seleccionaron 26 estudios para responder a la pregunta guía: “¿Puede el uso de medicamentos a base de plantas ayudar a controlar los signos y síntomas derivados del embarazo?", Continuando así la investigación. Después de leer los estudios, se identificó que 29 especies de plantas se utilizan como hierbas medicinales para el tratamiento de síntomas recurrentes en el embarazo, especialmente jengibre, manzanilla, menta y ajo. En cuanto a la prospección tecnológica, se seleccionaron seis patentes que, en su discusión, reportaron el potencial farmacológico del jengibre, la sandía, entre otros, en el tratamiento de síntomas comunes durante el embarazo. A partir de ello, se observa que a pesar del progresivo crecimiento de la importancia de las medicinas a base de plantas, se han realizado pocos estudios para comprobar la eficacia de su uso ante los síntomas característicos del período de gestación y que numerosas plantas medicinales son Aún aplicado desde el conocimiento bien empleado, enfatizando así la importancia del tema abordado.

Palabras clave: Atención primaria de salud; Cuidado prenatal; Fitoterapia; El embarazo.

\section{Introdução}

A gestação é um evento fisiológico que envolve transformações em vários aspectos tanto sociais, quanto físicos e emocional e deve ser vivenciado pelas gestantes como uma experiência de vida saudável e, a partir das modificações morfológicas e hormonais, fisiologicamente, o útero é o órgão mais passível de sofrer tais alterações, devido ao desenvolvimento fetal (Abreu, Lira Filho \& Santana, 2019).

Neste contexto, o tamanho e peso da cavidade uterina aumentam com o decorrer da gravidez e estas alterações fisiológicas desencadeiam modificações normais do funcionamento dos diversos sistemas, os quais originam desconfortos e sintomas apontados deste período, tendo o sistema digestório como um dos mais afetados pela variação hormonal, o que se confirma pelos frequentes episódios de náuseas, vômitos e azia neste período (Carvalho et al., 2016).

De acordo com Silva et al. (2015), durante o período gestacional, a necessidade de nutrientes aumenta para suprir o correto desenvolvimento fetal, assim como aumentam algumas queixas derivadas da gravidez, entretanto, apesar de não serem doenças, são condições que se tornam incômodas e, portanto, devem ser tratadas, podendo-se assim recorrer à fitoterapia.

As vantagens da fitoterapia na gestação são aderidas ao uso de medicamentos ou suplementos alimentares à base de plantas para aliviar esses desconfortos, agindo na prevenção dos sintomas e tratamento de complicações associadas a este período, tal como, o uso do gengibre e da camomila para o tratamento de distúrbios gastrointestinais anteriormente mencionados (Carvalho et al., 2016). 
A escolha dos fitoterápicos ocorre por uma série de fatores que vão desde a cultura popular, por acreditarem que as plantas não possuem efeitos prejudiciais à saúde, como também, pelas razões sociais, como o elevado preço dos medicamentos e a falta de acesso aos serviços de saúde (Lima et al., 2017). Segundo Camargo (2015), o uso de plantas medicinais é uma prática vinculada à medicina popular, isso adere a uma mistificação de conhecimentos da tradição local, mas também trazidos por outras culturas antigas, com a vantagem de ser uma medicina de fácil acesso e baixo custo relativo.

Nos últimos anos, a fitoterapia tem sido cada vez mais utilizada devido seus efeitos comprovados nos diversos sistemas do corpo humano. Estudos pré-clínicos realizados por Deepika et al. (2019) sugerem que há um interesse crescente em usar flavonóides, tais como os compostos bioativos, tendo em vista que são alternativas terapêuticas não tóxicas para tratar condições patológicas crônicas, incluindo o câncer.

Esse cenário mostra que terapias à base de plantas medicinais são amplamente utilizadas e, no Brasil, devido a sua maior biodiversidade, estimulou-se o uso de diferentes tipos de plantas, a exemplo da Myracrodruon urundeuva (aroeira-dosertão), pertecente à família Anacardiaceae. Seus compostos responsáveis pelos efeitos na prevenção, cura e tratamento de doenças são, especialmente, os antocianóides, flavononas, cumarinas, alcalóides, fitoesteróis, ácidos graxos, hidrocarbonetos, taninos, esteróides e os triterpenóides, como também, são relatados por suas atividades antimicrobianas contra bactérias, fungos e vírus, efeitos anti-inflamatórios, efeitos repelentes e larvicidas contra alguns mosquitos vetoriais (Yousaf \& Zuharah, 2015).

Dada a importância dos fitoterápicos para a redução dos sinais e sintomas apresentados pelas mulheres durante o período gravídico, tem-se como justificativa a elaboração dessa pesquisa como meio de levantar mais evidências científicas acerca desse tratamento. Para isso, utilizou-se como pergunta de investigação: o uso de fitoterápicos pode auxiliar no controle de sinais e sintomas advindos da gravidez? A partir da questão norteadora levantada, o objetivo geral do presente estudo foi avaliar, como base na literatura, a contribuição da fitoterapia para redução dos sinais e sintomas recorrentes durante a fase gestacional.

\section{Metodologia}

Trata-se de uma revisão integrativa de literatura, de cunho descritivo, com abordagem qualitativa, sustentando na identificação do problema levantado. Segundo Pereira et al. (2018), os aspectos metodológicos do estudo qualitativo possibilitam ao pesquisador, emitir opiniões próprias sobre o fenômeno de estudo interpretado.

Após elaborar a questão de investigação para o desenvolvimento da revisão integrativa por meio da estratégia PICO (População, Intervenção, Controle e Resultados), foram seguidos as seguintes etapas de elaboração: escolha do tema e formulação da questão norteadora, determinação dos critérios de inclusão e exclusão; definição dos descritores, busca na literatura, avaliação dos estudos incluídos na revisão integrativa a partir do tema retratado, análise crítica dos artigos e, por fim, a conclusão do estudo.

A busca da literatura foi executada no período de julho a novembro de 2020, utilizando as bases de dados relacionadas à área da saúde: ScienceDirect, National Library of Medicine (PUBMED), Literatura Latino-Americana e do Caribe em Ciências da Saúde (LILACS) e Scientific Electronic Library Online (SciELO).

A partir da delimitação dos Descritores em Ciências da Saúde (DeCS): "Atenção Primária à Saúde", "Cuidado PréNatal", "Fitoterapia" e "Gravidez", selecionou-se estudos que apresentassem em sua discussão, considerações relevantes acerca do uso da fitoterapia para a redução dos sinais e sintomas durante período gravídico, publicados nos últimos cinco anos, nos idiomas português, inglês e espanhol. Para tal, excluíram-se teses de doutorados, dissertações e trabalhos que não estavam na íntegra. 
Durante o período de levantamento das informações foram analisadas, também, patentes indexadas nas plataformas: Instituto Nacional da Propriedade Industrial (INPI), World Intellectual Property Organization (WIPO) e na Espacenet, publicadas entre os anos de 2010 a 2020 utilizando os descritores fitoterapia e gestação. Nas análises das bases de dados já mencionadas, o operador booleano "AND" foi utilizado para refinar a pesquisa.

Em seguida, organizou-se a amostra final por meio de um instrumento de coleta de dados, destacando-se: autores, ano de publicação, fitoterápicos, sinais e sintomas e os principais resultados encontrados após análise dos estudos. Por se tratar de uma revisão integrativa de literatura, não foi necessário submeter o estudo ao Comitê de Ética em Pesquisa.

\section{Resultados e Discussão}

Após triagem inicial nos bancos de dados foram encontrados 284 artigos científicos. Em seguida, foi realizada leitura rigorosa dos títulos e resumos que compuseram a pesquisa, excluindo-se, a partir disso, 22 estudos por estarem indexadas de forma simultânea em duas ou mais bases de dados e 236 por não atenderem aos critérios de inclusão. A amostra final foi composta por 26 estudos indexados nas plataformas propostas (Figura 1).

Figura 1 - Quantitativo de artigos científicos incluídos na revisão por base de dados.

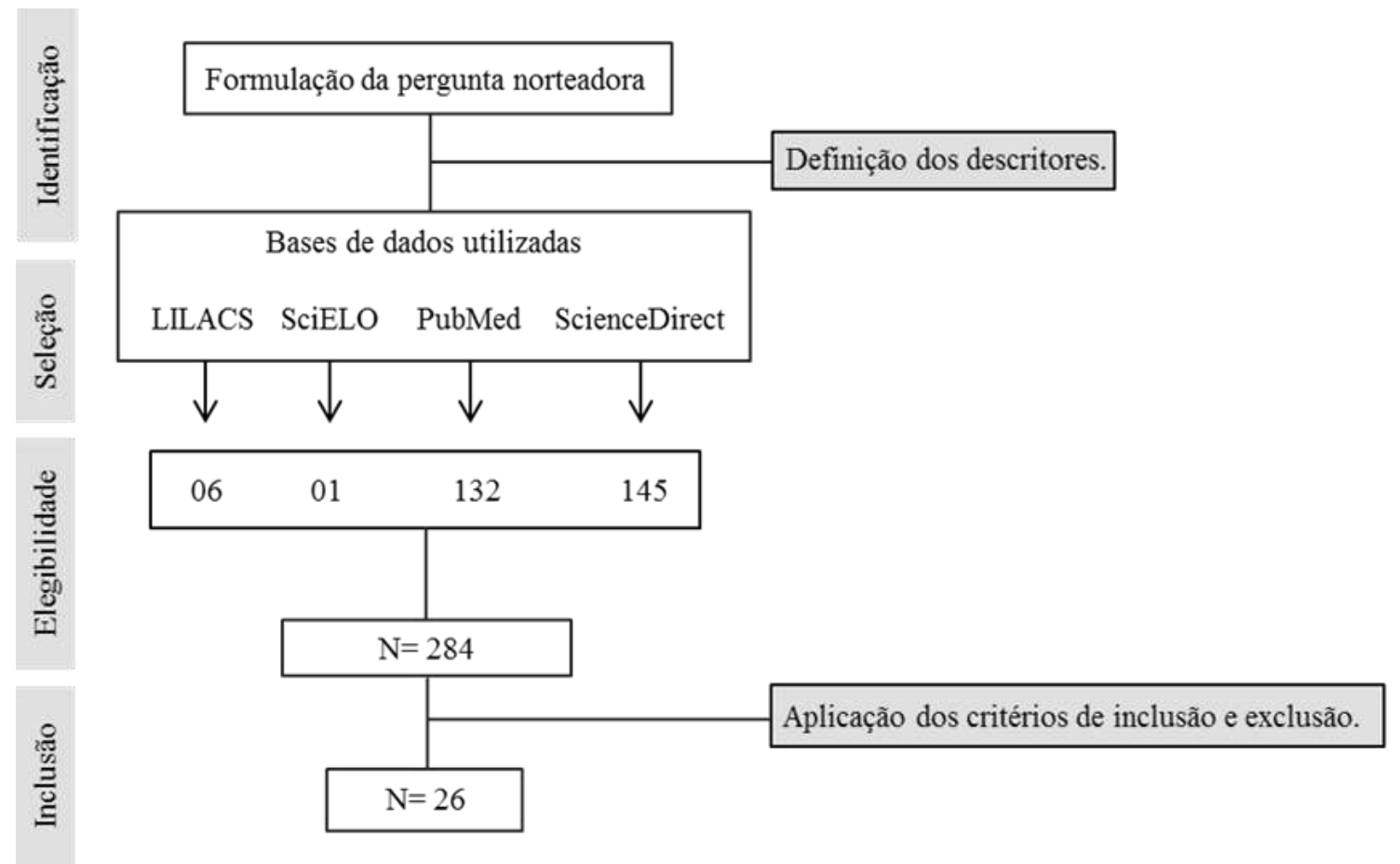

Fonte: Autoria própria (2020).

A distribuição dos estudos nas plataformas utilizadas demonstrou que 22 estudos se encontram simultaneamente na LILACS, PubMed e ScienceDirect. Na base de dados SciELO, apenas uma publicação foi selecionada por comtemplar os critérios de inclusão. Foram identificadas publicações, predominante, nos idiomas inglês (25) e português (1). Dos artigos selecionados, cinco foram publicados, respectivamente, em 2015, 2016 e 2018, quatro em 2017 e sete em 2019, ano com maior publicação científica sobre a temática abordada. Os principais resultados obtidos encontram-se estruturados na Tabela 1. 
Tabela 1 - Dados dos artigos selecionados relacionados aos fitoterápicos e seus benefícios.

\begin{tabular}{llllc}
\multicolumn{1}{c}{ Autores E Ano } & \multicolumn{1}{c}{ Fitoterápicos } & Sinais E Sintomas & \multicolumn{2}{c}{ Principais Resultados } \\
\hline LEWIS, E.; & $\begin{array}{l}\text { Linum usitatissimum } \\
\text { NICOLA, P. G. (2017). }\end{array}$ & Constipação. & $\begin{array}{l}\text { Apresentou } \\
\text { laxantes. }\end{array}$ & altas propriedades \\
\end{tabular}

\author{
AHMED, Seid M. et Zingiber officinale Roscoe \\ al., (2018). \\ (gengibre) Alliumsativum L. (alho) \\ Cucurbita pepo L. \\ (abobrinha).
}

BINA, F. et al., (2019). Pinus Pinaster (pinheiro-bravo).

YEMELE, M. D. et al., Cymbopogon citratus (capim(2015). santo), Sida veronicifolia (malva branca) e Nelsonia canescens.

BARNES, L. A. J. et al., Oenothera biennis (prímula). (2019).

ELMAZOUDY, R. H.; Zingiber officinale Roscoe ATTIA, A. A. (2018). (gengibre).

CORROTO, F. TORRES G.; MACÍA, (2019). O. A. Mollis (munha) Matricaria M. J. recutita (camomila-vulgar)

CATARINO, L.; Carica papaya L (mamão). HAVIK, P. J.; ROMEIRAS, M. M. (2016).

TAVAKOLY, R. et al., (2018).

CARDOSO, B. S.; Chamomile, (camomila). Zingiber AMARAL, V. C. S. (gengibre); (2019).
Minthostachys

Trigonella foenum-graecum (sementes de feno-grego).

Náuseas e Êmeses.

Inflamação.

Edema e dores.

Pele ressecada,

Náuseas e vômitos.

Distúrbios

intestinais.

Distúrbios intestinais.

Inflamação.

Náuseas e Êmeses; inflamação; flatulência, cefaleia Mentha $x$ villosa, (menta e e mialgias. hortelã); Echinacea purpúrea, Allium sativum (alho).

\begin{abstract}
KOOHPAYEHZADEH, J.; REZAIAN, F.; SHEIKHANSARI, N.; ESHRAGHI, N. (2018).
\end{abstract}

SHARIFZADEH, F.; Zingiber KASHANIAN, M.; (gengibre).

BOLTMANBINKOWSKI, (2016). officinale Roscoe Náuseas e êmese.

Zingiber,

H. (gengibre)
Mostrou ser um forte aliviante e foram relatados poucos efeitos adversos.

Apresentou forte ação teraprêutica frente a dores na gravidez e endometriose associada.

Apresentou forte ação antiedematosa. Demonstrou efeitos analgésicos, diuréticos $\mathrm{e}$ antimaláricos.

Apresentou melhora da hidratação da pele.

Mostrou ser um forte antiemético e, quando utilizado em altas concentrações podem causar retardo do crescimento fetal.

Essas plantas apresentaram ação farmacológica no sistema digestivo.

Seu uso destaca-se: na gravidez, parto, amamentação e cuidados infantis.

Apresentou propriedades digestivas e anti-inflamatórias.

A camomila e o gengibre apresentaram fortes atividades antieméticas; a menta e a hortelã mostraram-se eficazes em distúrbios gástricos, dispepsia, cefaleia e mialgia; já o alho apresentou ação anti-inflamatória.

Apresentou forte ação antiemética

Não apresentou efeitos teratogênicos. 
TRABACE, L.; TUCCI, P.; CIUFFREDA, L. et al., (2015).
Matricaria chamomilla

(camomila); Pimpinella anisum (erva-doce), Melissa officinalis (rrva-cidreira), Zingiber officinale Roscoe (gengibre), Valeriana officinalis (valeriana) e Malva sylvestris (malva).

BALBONTÍN, Y. M.; Zingiber officinale Roscoe STEWART, D.; (gengibre). SHETTY, A.; FITTON, C. A.; MCLAY, J. S. (2019).

MCLAY, J. S.; IZZATI, N.; PALLIVALAPIL,A. A. R. et al., (2017).

AKOUR, A.; Matricaria aurea (camomila)

KASABRI, V.; AFIFI, F. U.; BULATOVA, N. (2016).

IZZO, A. A; HOONKIM, $\quad$ S.; RADHAKRISHNAN,

R.; WILLIAMSON E.

M. (2016).

POUR, Z. S.; HOSSEINKHANI, A.; ASADI, N. et al., (2018).

NEGA, S. S.; BEKELE, Ocimum lamiifolium (manjericão) H. M.; MELES, G. G.; NORDENG, H. (2019).

ALI-SHTAYEH M. S.; Pimpinella anisum JAMOUS, R. M.; (erva-doce), Salvia fruticosa, JAMOUS, R. M. Matricaria aurea (camomila) e (2015).

Mentha spicata (hortelã-verde).

PEPRAH, P.; Zingiber officinale (gengibre), AGYEMANG-DUAH, Mentha-piperita,

W.; $\quad$ ARTHUR- (hortelã-pimenta),

Salvia

HOLMES, F. et al., officinalis (sálvia), Allium sativum (2019). (alho), Echinacea purpúrea (folha de echinacea).

NERGARD, C. S.; HO, Lippia chevalieri, Combretum T. P.; DIALLO, D.; micranthum (kinkeliba no Benin), BALLO, N.; Parkia biglobosa (alfarroba) e PAULSEN, B. S.; Vepris heterophylla.

NORDENG, H. (2015).

KENNEDY, D. A; Zingiber officinale (gengibre), LUPATTELLI, A.; Vaccinium oxycoccus KOREN, G.; macrocarpon (cranberry) e NORDENG, H. (2016). Mentha - piperita (hortelãpimenta).

$\begin{array}{llr}\begin{array}{l}\text { Distúrbios } \\ \text { gastrintestinais. }\end{array} & \begin{array}{l}\text { Relatou } \\ \text { fitoterápicos }\end{array} \\ & \begin{array}{l}\text { tomados por via oral e durante todo } \\ \text { o período da gravidez. }\end{array}\end{array}$

Náusea e vômitos.

Quatorze estudos relatou ausência de eventos adversos decorrente deles.

Insônia, náuseas e vômitos, problemas gastrintestinais.

Mostrou um maior potencial do gengibre em relação à camomila quanto a sua ação antiemética.

Náuseas e vômitos.

Uso para náusea e vômitos no início da gravidez ou em todo gravidez

Náuseas e vômitos. Demostrou reduzir a náusea induzida pela gravidez.

Insônia.
Resfriado cefaleia.

Distúrbios abdominais, constipação resfriado.

Vômito e náusea, resfriado, dor nas costas, tontura, estresse, depressão resfriado.

Edema

Distúrbios gastrointestinais febre,

e
Sugerem que as sementes de alface diminuíram a insônia durante a gravidez e podem ser recomendadas como remédio natural seguro para o tratamento da insônia na gravidez.

e Mostrou eficácia para uso comum em resfriado e cefaleia durante a gravidez.

Uso durante todas as fases da gravidez não há riscos para saúde.

$\mathrm{O}$ gengibre mostrou eficaz no tratamento de muitas formas de náusea, folhas de salvia e echinacea fornecem tratamento natural para aliviar ou curar a depressão, e que hortelã e alho são eficazes contra resfriado comum.

Melhora e reduz o edema.

Demostrou serem ervas seguras no tratamento de distúrbios gastrointestinais. 


\begin{tabular}{|c|c|c|c|}
\hline $\begin{array}{l}\text { FRAWLEY, J. et al., } \\
(2015) \text {. }\end{array}$ & $\begin{array}{l}\text { Matricaria chamomilla } \\
\text { (camomila), Zingiber officinale } \\
\text { (gengibre). }\end{array}$ & $\begin{array}{l}\text { Insônia, náusea e } \\
\text { vômito. }\end{array}$ & $\begin{array}{l}\text { O uso da camomila auxilia no } \\
\text { tratamento da isônia. Os resultados } \\
\text { mostram um aumento uso de } \\
\text { fitoterápicos por mulheres com } \\
\text { fadiga. }\end{array}$ \\
\hline $\begin{array}{l}\text { KISSAL, A.; ÇEVIK } \\
\text { GÜNER Ü, BATKIN } \\
\text { ERTÜRK, D. (2017). }\end{array}$ & $\begin{array}{l}\text { Tilia (linden), Mentha - piperita } \\
\text { (hortelã-pimenta), Citrus limon } \\
\text { (limão) e Zingiber officinale } \\
\text { (gengibre). }\end{array}$ & Gripe e resfriado. & $\begin{array}{l}\text { Mostrou o uso devido comum } \\
\text { durante gravidez no } 1^{\mathrm{o}} \text { e } 2^{\circ} \\
\text { trimestre. }\end{array}$ \\
\hline $\begin{array}{l}\text { JAMES, P. B.; BAH, A. } \\
\text { J.; TOMMY, M. S.; } \\
\text { WARDLE, J.; STEEL, } \\
\text { A. (2018). }\end{array}$ & $\begin{array}{l}\text { Luffa acutangula } \\
\text { Roxb (bucha-de-purga } \\
\text { abrobinha do norte). }\end{array}$ & $\begin{array}{l}\text { Infecção do trato } \\
\text { urinário e edema } \\
\text { do pedal. }\end{array}$ & $\begin{array}{l}\text { Indicado principalmente para } \\
\text { infecção do trato urinário e edema } \\
\text { do pedal. }\end{array}$ \\
\hline
\end{tabular}

Fonte: Autoria própria (2020).

No que se refere às ervas utilizadas, apenas as espécies Zingiber officinale (gengibre), Matricaria chamomilla (camomila), Mentha spicata (hortelã), Allium sativum (alho), Citrus limon (limão) e Pimpinella anisum (erva-doce) se repetiram nos estudos (Figura 2), destacando-se como os mais citados o gengibre (25\%), seguido da camomila $(12,5 \%)$ e hortelã $(8,9 \%)$.

Figura 2 - Representação dos fitoterápicos utilizados nos artigos por frequência de citações.

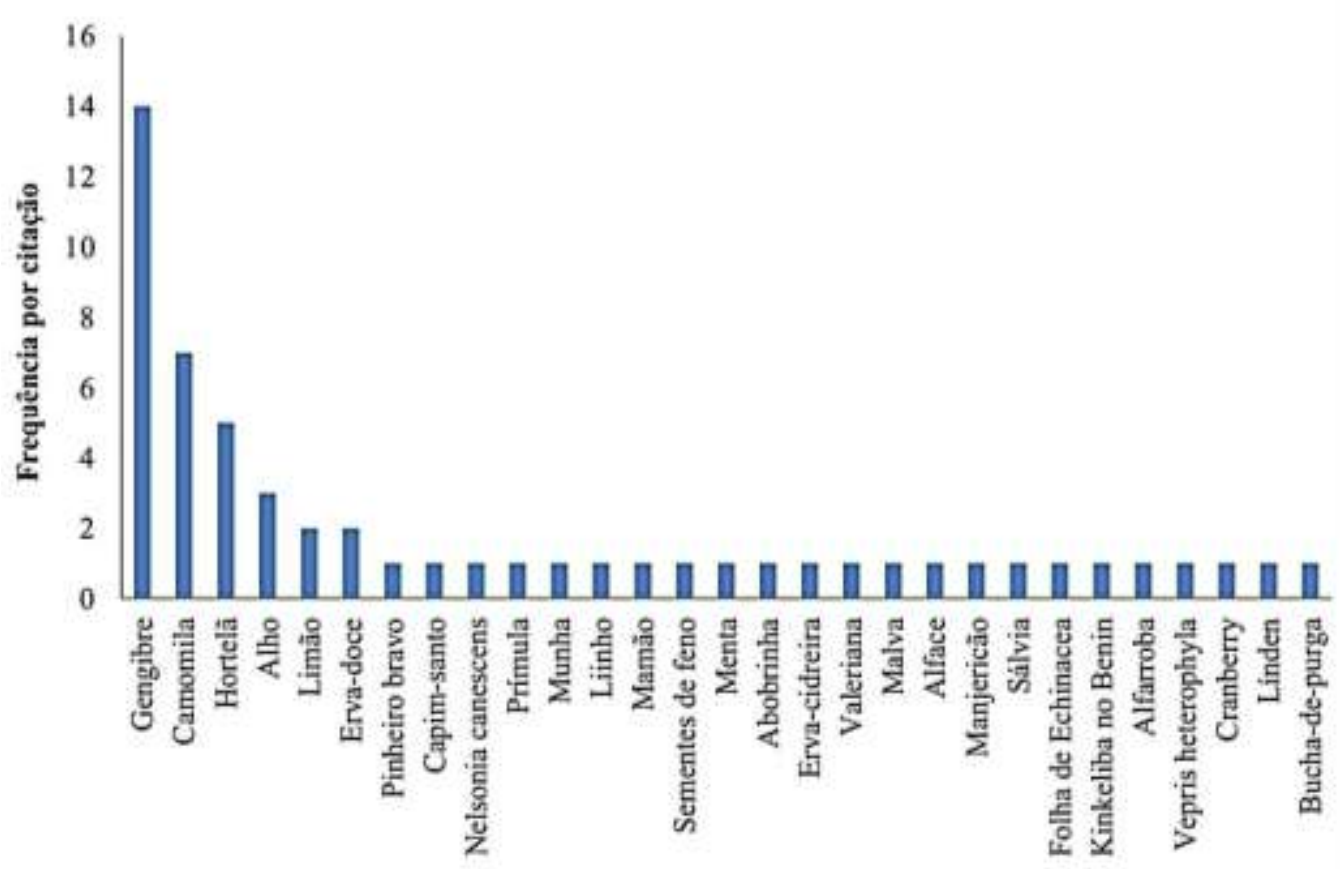

Fitoterápicos utilizados

Fonte: Autoria própria (2020).

O Zingiber officinale (gengibre) e a Matricaria chamomilla (camomila), como o mais citados em relação aos demais fitoterápicos, respectivamente, se mostraram altamente eficientes em todos os estudos e com um poderoso poder de resolutividade frente a problemas gastrointestinais, mais especificamente relacionados a náuseas e vômitos, como também demonstraram fortes atividades anti-insônia (Cardoso \& Amaral, 2019; Ahmed et al., 2018; Kennedy, Lupattelli, Koren \& Nordeng, 2016; Frawley et al., 2015). 
O gengibre, além de suas ações antiemética e anti-insônia também apresentou atividade antigripal (Frawley et al., 2015). Contudo, no estudo realizado por Elmazoudy et al. (2018), em camundongos, relatou-se que seu uso em altas concentrações resulta em retardo do crescimento do corpo lúteo por insuficiência do hormônio progesterona durante o início da gravidez. No entanto, de acordo com pesquisas realizadas mais recentemente por Nega et al. (2019), o gengibre pode ser utilizado ao decorrer de toda a gravidez não havendo evidências de riscos, ou seja, não causando ações teratogênicas ao binômio.

Com relação às espécies com menor frequência no estudo (Figura 2), algumas como: Valeriana officinalis (valeriana), Luffa acutangula (L.) Roxb (bucha-de-purga), Lippia chevalieri, Combretum micranthum (kinkeliba no Benin), Parkia biglobosa (alfarroba), Echinacea purpúrea, folha de echinacea, Vepris heterophylla, Oenothera biennis (prímula) e Nelsonia canescens são pouco conhecidas, entretanto, com fortes ações fitoterápicas frente ao edema, cefaleia, inflamações, distúrbios gastrointestinais e, até mesmo, contra a depressão, como as folhas de echinacea (Cardoso \& Amaral, 2019; James et al., 2018; Nergard et al., 2015; Trabace et al., 2015).

Em concordância, foram relatadas algumas espécies bastante conhecidas como o Citrus limon (limão), a Pimpinella anisum (erva-doce), o Ocimum lamiifolium (manjericão), o Lactuca Sativa L. (alface), a Melissa officinalis (erva-cidreira), o Carica papaya L. (mamão), as quais, apesar de serem comuns e promoverem inúmeros benefícios como ação antigripal, calmante e anti-insônia, também agem contra distúrbios gastrointestinais, no entanto, estudos voltados aos benefícios frente aos sintomas gravídicos ainda são escassos na literatura (Nega, Bekele, Meles \& Nordeng, 2019; Pour et al., 2018; Trabace et al., 2015; Ali-shtayeh, Jamous \& Jamous, 2015).

Embora o gengibre e a camomila tiverem apresentado maiores incidências em relação aos demais, alguns outros fitoterápicos também se destacaram por apresentarem ações farmacológicas relevantes frente aos sinais e sintomas característicos do período gestacional, conforme representado na figura 3.

Figura 3 - Representação da frequência dos sinais e sintomas por artigos avaliados.

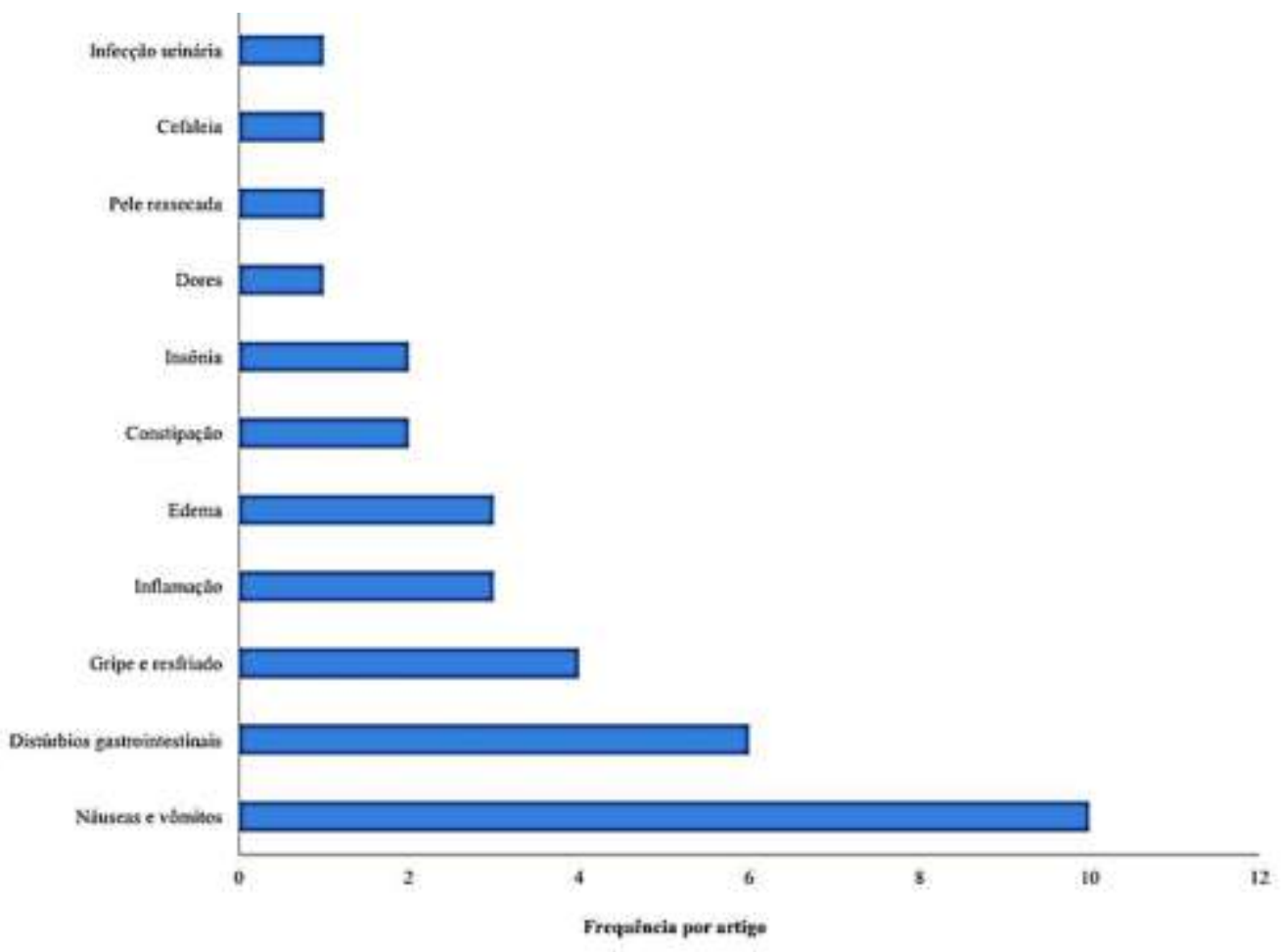

Fonte: Autoria própria (2020). 
Comparando-se com os sintomas de náuseas e vômitos, indicaram-se na Figura 3, os sintomas característicos da gestação, porém menos frequentes, como o edema, insônia, pele ressecada, inflamação, gripe e resfriado, distúrbios gastrointestinais, cefaleia, dores e infecção urinária. É importante ressaltar que alguns desses sintomas são relatados corriqueiramente por muitas gestantes, a exemplo, constipação, edema e infecção urinária, mas que apresentaram uma menor frequência entre os estudos avaliados, mostrando, desse modo, a importância de novas publicações científicas que abordem a utilização de fitoterápicos diante desses sintomas.

De acordo com os resultados obtidos, apenas um artigo abordou a utilização de Luffa acutangula (L.) Roxb (bucha-depurga) como fitoterápico para o tratamento de infecções do trato urinário, destacando sua eficácia frente ao edema de pedal (James et al., 2018). No entanto, apenas dois artigos abordaram o uso de plantas para o tratamento dos sintomas de constipação. Elise \& Nicola (2017), realizaram seu estudo com o Linum usitatissimum (linho), por conter altas propriedades laxantes. Ademais, Ali-Shtayeh et al. (2015) utilizaram as espécies Pimpinella anisum (erva-doce), Salvia fruticosa (salvia), Matricaria aurea (camomila) e Mentha spicata (hortelã-verde) e obtiveram resultados de que seu uso durante todas as fases da gravidez não evidenciaria riscos para a saúde posteriormente.

A respeito dos sintomas característicos de insônia, Marques et al. (2011) evidenciaram que episódios de insônia são frequentes devido às alterações hormonais, emocionais, mentais e físicas. Comparando-se com pesquisas de Pour et al. (2018), foi avaliado que o uso das sementes de alface frente ao distúrbio do sono, diminuíram a insônia durante a gravidez e pode ser recomenda como remédio natural seguro para o tratamento da insônia relacionada à gravidez, ressaltando-se, também, como um poderoso fitoterápico e pouco abordado nas literaturas atuais.

Ademais, a cefaleia, pele ressecada, infecção, dores, gripes e resfriados, também são sintomas bastante comuns nesse período e criam um incômodo aparente. No que tange aos fitoterápicos utilizados, destacaram-se o limão, a gengibre e a hortelã, para quadros gripais, os quais são muito utilizados durante a gravidez no $1^{\circ}$ e $2^{\circ}$ trimestre (Kissal, Güner \& Ertürk, 2017), assim como, o manjericão, capim-santo e o pinheiro-bravo para dores e cefaleia (Nega, Bekele, Meles \& Nordeng, 2019; Bina et al., 2019; Yemele et al., 2015) e a prímula, indicada para pele ressecada (Barnes et al., 2019).

A partir das análises realizadas nas plataformas de patentes anteriormente mencionadas, não foram encontradas informações na WIPO e INPI, dessa forma, utilizou-se apenas a Espacenet por apresentar resultados dentro dos padrões estabelecidos para esta prospecção, e encontram-se abaixo na Tabela 2.

Tabela 2 - Representação das patentes analisadas publicadas entre os anos de 2010 a 2020 (Espacenet).

\begin{tabular}{lll}
\hline \multicolumn{1}{c}{ ID } & \multicolumn{1}{c}{ TÍTULO } & CÓDIGO \\
\hline PT1 & Composition comprising herbal extracts & WO2020079682A1 \\
PT2 & Nutritional composition containing ginger extract for enhancing tight & KR20180009868A \\
& junctions in intestinal cells and prepeparing method thereof & \\
PT3 & Phytotea "avan" & RU2604787C1 \\
PT4 & Ginger extract for the protection of stem cells & CN104000752A \\
PT5 & Diuretic agente & RU2442598C1 \\
PT6 & Antihyperglycemic and anti-obesity active herbal compound & BRPI0905103A2 \\
\hline
\end{tabular}

Legenda: ID - Nomenclatura sugerida para facilitar a identificação das patentes.

Fonte: Autoria própria (2020).

As seis patentes (Tabela 2) referem-se ao uso de fitoterápicos para tratar e/ou prevenir sinais e sintomas comuns na gravidez. A PT1 relata que as espécies Terminalia chebula e Tinospora cordifolia possuem inúmeras atividades farmacológicas já evidenciadas cientificamente, frente a episódios de diarreia e vômitos e aos quadros de resfriado. Por sua 
vez, a PT2, PT3 e PT5 relataram, sobretudo, quanto à utilização do gengibre (Zingiber officinale) no tratamento da hiperpigmentação da pele (cloasma gravídico) e ao seu potencial efeito nos distúrbios gastrointestinais, também mencionados nos estudos de Cardoso e Amaral (2019), Carvalho et al. (2016) e Frawley et al. (2015).

Em relação a PT4 e PT6, os autores abordaram a ação diurética do extrato das folhas de Birch e da melancia (Citrullus lanatus), os quais evidenciaram um aumento na produção de urina e boa ação em relação aos parênquimas renais. Na PT7, por sua vez, produziram o extrato aquoso e hidroalcoólico da espécie Plathymenia sp, o que resultou em um composto ativo antihiperglicêmico e anti-obesidade.

Quanto a Classificação Internacional de Patentes (IPC/CIP), o código que mais se repetiu foi o A61K36, o qual está relacionado a preparações medicinais de constituição indeterminada contendo material de algas, líquenes, fungos ou plantas e seus derivados, de acordo com WIPO. Por fim, mediante os resultados apresentados, nota-se que a utilização de plantas medicinais como agentes fitoterápicos vem crescendo cada vez mais em virtude dos benefícios, fácil acesso e do baixo custo relativo a essa terapêutica.

\section{Conclusão}

A análise realizada nesta revisão mostrou as espécies de fitoterápicos mais utilizados por consideração do crescimento progressivo e da importância deles na vivência cotidiana durante a gestação. Os resultados obtidos demonstraram haver uma maior prevalência da utilização do Zingiber officinale (gengibre), da Matricaria chamomilla (camomila), da Mentha-piperita (hortelã) e do Allium sativum (alho) em episódios de náuseas e vômitos, distúrbios gastrointestinais, síndromes gripais e edemas, sintomas estes bem característicos do período gestacional.

Ainda que haja estudos patenteados acerca da utilização de espécies vegetais diante dos problemas levantados, verificou-se a escassez de pesquisas na literatura que abordassem a eficácia da utilização de produtos naturais frente aos sintomas característicos do período gravídico, independentemente de variáveis geográficas, socioeconômicas e étnico-culturais que eventualmente possam distingui-las. Além disso, como o Brasil tem uma grande diversidade de plantas, foi perceptível que há inúmeras espécies que possuem ações fitoterápicas e deixam de ser utilizadas a nível mundial por falta de conhecimento a respeito dos benefícios reais que estas podem oferecer, dando assim, subsídios à continuidade do trabalho.

Desse modo, sugere-se que pesquisas futuras direcionem estudos voltados aos principais efeitos dos fitoterápicos no tratamento de sinais e sintomas recorrentes da gravidez, bem como, ao correto uso de medicamentos e suplementos a base de fitoterápicos, tendo em vista que, atualmente, essas plantas medicinais ainda são utilizadas por uma grande parcela da população, sobretudo, por apresentarem efeitos profiláticos, medicamentosos e paliativos, sendo aplicado a partir do conhecimento popular empregado de modo a desmistificar a ideia de que produtos de origem natural são ofensivos e prejudiciais à saúde, ressaltando assim, a relevância do presente estudo.

\section{Referências}

Abreu da Silva, A. C., \& Botelho de Santana, L. L. (2018). Os riscos do uso de plantas medicinais durante o período gestacional: uma revisão bibliográfica. Acta toxicol. argent, 118-123.

Abreu, L. P., Lira Filho, R., \& de Santana, R. L. (2019). Características obstétricas das gestantes submetidas à cesariana segundo a Classificação de Robson [Obstetric characteristics of pregnant women undergoing cesarean section, by the Robson Classification]. [Características obstétricas de las mujeres embarazadas sometidas a cesárea según la Clasificación de Robson]. Revista Enfermagem UERJ, $27,37858$.

Ahmed, S. M., Nordeng, H., Sundby, J., Aragaw, Y. A., \& de Boer, H. J. (2018). The use of medicinal plants by pregnant women in Africa: A systematic review. Journal of ethnopharmacology, 224, 297-313.

Akour, A., Kasabri, V., Afifi, F. U., \& Bulatova, N. (2016). The use of medicinal herbs in gynecological and pregnancy-related disorders by Jordanian women: a review of folkloric practice vs. evidence-based pharmacology. Pharmaceutical Biology, 54(9), 1901-1918. 
Ali-Shtayeh, M. S., Jamous, R. M., \& Jamous, R. M. (2015). Plants used during pregnancy, childbirth, postpartum and infant healthcare in Palestine. Complementary Therapies in Clinical Practice, 21(2), 84-93.

Balbontín, Y. M., Stewart, D., Shetty, A., Fitton, C. A., \& McLay, J. S. (2019). Herbal medicinal product use during pregnancy and the postnatal period: a systematic review. Obstetrics and gynecology, 133(5), 920.

Barnes, L. A., Barclay, L., McCaffery, K., \& Aslani, P. (2019). Complementary medicine products: information sources, perceived benefits and maternal health literacy. Women and Birth, 32(6), 493-520.

Bina, F., Soleymani, S., Toliat, T., Hajimahmoodi, M., Tabarrai, M., Abdollahi, M., \& Rahimi, R. (2019). Plant-derived medicines for treatment of endometriosis: a comprehensive review of molecular mechanisms. Pharmacological research, 139, 76-90.

Boltman-Binkowski, H. (2016). A systematic review: Are herbal and homeopathic remedies used during pregnancy safe? Curationis, 39(1), 1-8.

Braun, V., \& Clarke, V. (2006). Utilizando el análisis temático en psicología. Investigación cualitativa en psicología, 3(2), 77-101.

Camargo, F. R. (2015). Promoção da saúde Materno-Infantil: grupo reflexivo sobre o uso de plantas medicinais e medicamentos fitoterápicos na gravidez e lactação.

Cardoso, B. S., \& Amaral, V. C. S. (2019). O uso da fitoterapia durante a gestação: um panorama global. Ciência \& Saúde Coletiva, 24, 1439-1450.

Carvalho, C. S. C. D. (2016). Fitoterapia na gravidez: segurança e eficácia de produtos à base de plantas no alívio de sintomas e desconfortos associados à gravidez.

Catarino, L., Havik, P. J., \& Romeiras, M. M. (2016). Medicinal plants of Guinea-Bissau: Therapeutic applications, ethnic diversity and knowledge transfer. Journal of Ethnopharmacology, 183, 71-94.

Corroto, F., Gamarra Torres, O. A., \& Macía, M. J. (2019). Different patterns in medicinal plant use along an elevational gradient in northern Peruvian Andes. Journal of ethnopharmacology, 239, 111924.

Deepika, M. S., Thangam, R., Sheena, T. S., Sasirekha, R., Sivasubramanian, S., Babu, M. D., \& Thirumurugan, R. (2019). A novel rutin-fucoidan complex based phytotherapy for cervical cancer through achieving enhanced bioavailability and cancer cell apoptosis. Biomedicine \& Pharmacotherapy, 109, 11811195 .

Duarte, A. F. S., da Costa Martins, A. L., Miguel, M. D., \& Miguel, O. G. (2018). O uso de plantas medicinais durante a gravidez e amamentação. Visão Acadêmica, 18(4).

ElMazoudy, R. H., \& Attia, A. A. (2018). Ginger causes subfertility and abortifacient in mice by targeting both estrous cycle and blastocyst implantation without teratogenesis. Phytomedicine, 50, 300-308.

Fereday, J., \& Muir-Cochrane, E. (2006). Demonstrating rigor using thematic analysis: A hybrid approach of inductive and deductive coding and theme development. International journal of qualitative methods, 5(1), 80-92.

Frawley, J., Adams, J., Steel, A., Broom, A., Gallois, C., \& Sibbritt, D. (2015). Women's use and self-prescription of herbal medicine during pregnancy: an examination of 1,835 pregnant women. Women's Health Issues, 25(4), 396-402.

Izzo, A. A., Hoon-Kim, S., Radhakrishnan, R., \& Williamson, E. M. (2016). A critical approach to evaluating clinical efficacy, adverse events and drug interactions of herbal remedies. Phytotherapy Research, 30(5), 691-700.

James, P. B., Bah, A. J., Tommy, M. S., Wardle, J., \& Steel, A. (2018). Herbal medicines use during pregnancy in Sierra Leone: An exploratory crosssectional study. Women and Birth, 31(5), e302-e309.

Kennedy, D. A., Lupattelli, A., Koren, G., \& Nordeng, H. (2016). Safety classification of herbal medicines used in pregnancy in a multinational study. BMC Complementary and Alternative Medicine, 16(1), 1-9.

Kıssal, A., Güner, Ü. Ç., \& Ertürk, D. B. (2017). Use of herbal product among pregnant women in Turkey. Complementary therapies in medicine, $30,54-60$.

Lewis, E., \& Glover, P. N. (2017). Avaliação de segurança de medicamentos fitoterápicos na gravidez. Toxicologia Reprodutiva, 72, 16.

McLay, J. S., Izzati, N., Pallivalapila, A. R., Shetty, A., Pande, B., Rore, C., \& Stewart, D. (2017). Pregnancy, prescription medicines and the potential risk of herb-drug interactions: a cross-sectional survey. BMC Complementary and Alternative Medicine, 17(1), 1-7.

Lima, R., Turrini, R., Silva, L. R., Melo, L., \& Augusto, S. I. (2017). Popular healing practices and medical plants use for riparian mothers in early childhood care Práticas populares de cura e o uso de plantas medicinais por mães ribeirinhas no cuidado infantil. Revista de Pesquisa: Cuidado é Fundamental Online, 9(4), 1154-1163.

McParlin, C., O'Donnell, A., Robson, S. C., Beyer, F., Moloney, E., Bryant, A., \& Vale, L. (2016). Treatments for hyperemesis gravidarum and nausea and vomiting in pregnancy: a systematic review. Jama, 316(13), 1392-1401.

Nega, S. S., Bekele, H. M., Meles, G. G., \& Nordeng, H. (2019). Medicinal plants and concomitant use with pharmaceutical drugs among pregnant women. The Journal of Alternative and Complementary Medicine, 25(4), 427-434.

Nergard, C. S., Ho, T. P. T., Diallo, D., Ballo, N., Paulsen, B. S., \& Nordeng, H. (2015). Attitudes and use of medicinal plants during pregnancy among women at health care centers in three regions of Mali, West-Africa. Journal of Ethnobiology and Ethnomedicine, 11(1), 1-11. 
Research, Society and Development, v. 10, n. 3, e11610313065, 2021

(CC BY 4.0) | ISSN 2525-3409 | DOI: http://dx.doi.org/10.33448/rsd-v10i3.13065

Peprah, P., Agyemang-Duah, W., Arthur-Holmes, F., Budu, H. I., Abalo, E. M., Okwei, R., \& Nyonyo, J. (2019). 'We are nothing without herbs': a story of herbal remedies use during pregnancy in rural Ghana. BMC Complementary and Alternative Medicine, 19(1), 1-12.

Pereira A.S. et al. (2018). Metodologia da pesquisa científica. UFSM. https://repositorio.ufsm.br/bitstream/handle/1/15824/Lic_Computacao_MetodologiaPesquisa-Cientifica.pdf?sequence=1.

Pitilin, É. D. B., \& Pelloso, S. M. (2017). Internações sensíveis à atenção primária em gestantes: fatores associados a partir do processo da atenção pré-natal. Texto \& Contexto-Enfermagem, 26(2).

Pour, Z. S., Hosseinkhani, A., Asadi, N., Shahraki, H. R., Vafaei, H., Kasraeian, M., \& Faraji, A. (2018). Double-blind randomized placebo-controlled trial on efficacy and safety of Lactuca sativa L. seeds on pregnancy-related insomnia. Journal of ethnopharmacology, 227, 176-180.

Sharifzadeh, F., Kashanian, M., Koohpayehzadeh, J., Rezaian, F., Sheikhansari, N., \& Eshraghi, N. (2018). A comparison between the effects of ginger, pyridoxine (vitamin B6) and placebo for the treatment of the first trimester nausea and vomiting of pregnancy (NVP). The Journal of Maternal-Fetal \& Neonatal Medicine, 31(19), 2509-2514.

Silva, M. G. D. (2015). Plantas medicinais na gravidez e aleitamento.

Tavakoly, R., Maracy, M. R., Karimifar, M., \& Entezari, M. H. (2018). Does fenugreek (Trigonella foenum-graecum) seed improve inflammation, and oxidative stress in patients with type 2 diabetes mellitus? A parallel group randomized clinical trial. European Journal of Integrative Medicine, 18, 13-17.

Trabace, L., Tucci, P., Ciuffreda, L., Matteo, M., Fortunato, F., Campolongo, P., \& Cuomo, V. (2015). "Natural” relief of pregnancy-related symptoms and neonatal outcomes: above all do no harm. Journal of ethnopharmacology, 174, 396-402.

Yemele, M. D., Telefo, P. B., Lienou, L. L., Tagne, S. R., Fodouop, C. S. P., Goka, C. S., \& Moundipa, F. P. (2015). Ethnobotanical survey of medicinal plants used for pregnant women's health conditions in Menoua division-West Cameroon. Journal of Ethnopharmacology, 160, 14-31.

Yousaf, A., \& Zuharah, W. F. (2015). Lethal response of the dengue vectors to the plant extracts from family Anacardiaceae. Asian Pacific Journal of Tropical Biomedicine, 5(10), 812-818. 